\title{
Zinc and ageing: third Zincage conference Eugenio Mocchegiani
}

\author{
Address: Immunolgy Ctr. (Section Nutrition, Immunity and Ageing) Res. Dept. INRCA, Ancona, Italy
}

Email: Eugenio Mocchegiani - e.mocchegiani@inrca.it

Published: 20 September 2007

Immunity \& Ageing 2007, 4:5 doi: 10.1 I86/1742-4933-4-5

This article is available from: http://www.immunityageing.com/content/4/I/5

(c) 2007 Mocchegiani; licensee BioMed Central Ltd.

This is an Open Access article distributed under the terms of the Creative Commons Attribution License (http://creativecommons.org/licenses/by/2.0), which permits unrestricted use, distribution, and reproduction in any medium, provided the original work is properly cited.
Received: II July 2007

Accepted: 20 September 2007

\begin{abstract}
The importance of $\mathrm{Zn}$ for optimal functioning of the immune system and antioxidant stress response is well documented. $\mathrm{Zn}$ homeostasis influences development and function of immune cells, activity of stress-related and antioxidant proteins [metallothioneins (MT), chaperones, Apo], Poly(ADP-Ribose) polymerase-I (PARP-I) and Methionione Sulfoxide Reductase (Msr), Superoxide Dismutase (SOD)], and helps to maintain genomic integrity and stability. During ageing, the intake of $\mathrm{Zn}$ decreases due to inadequate diet and/or intestinal malabsorption, contributing to frailty, general disability and increased incidence of age-related degenerative diseases (cancer, infections and atherosclerosis). Although many factors contributing to $\mathrm{Zn}$ deficiency have been identified, the biochemical markers of $Z n$ deficiency as well as the possibility to achieve relevant health benefits through $\mathrm{Zn}$ supplementation in the elderly are still a matter for evaluation. Taking into account that $\mathrm{Zn}$ homeostasis is regulated by proteins and enzymes for which polymorphisms have been previously found to be associated with successful/unsuccessful ageing, genetic screening might be of added value in evaluating the individual response to $\mathrm{Zn}$ supplementation. Biochemical, immunological, dietary and genetic studies aimed at understanding the impact of $\mathrm{Zn}$ in healthy ageing, the effect of $Z n$ supplementation in the elderly and finally formulating a rationale for the promotion of correct $\mathrm{Zn}$ supplementation were discussed at the international Zincage conference held in Ancona in January 2007.
\end{abstract}

\section{Introduction}

Zincage [1] is a specific targeted research project (STREP) funded by the European Union in the 6th Framework Program (FP6). It includes epidemiological studies on the influence of diet and lifestyle on healthy ageing, aimed at preventing adult degenerative disease, particularly focusing on cardiovascular diseases and also addressing malnutrition of the elderly. The conference held in Ancona, January 2007, was focussed particularly on the effects of $\mathrm{Zn}$ supplementation in the elderly and on the possible influences of dietary, biochemical and genetic factors on the individual response. $\mathrm{Zn}$ deficiency, cell-mediated immune dysfunction and increased oxidative stress are common in elderly subjects and it is quite clear that die- tary habits including Zn consumption have a great impact on these factors. Zn supplementation in the elderly can improve the immune response and reduce oxidative stress markers, thereby contributing to a reduced incidence of infections. However, individual differences in the response to $\mathrm{Zn}$ can lead to contradictory results even with supplementation trials performed in elderly people of the same age-groups. One of the reasons for these individual differences is the different genetic background of the subjects enrolled in the study. In fact, some proteins with documented polymorphic sites are involved in regulating $\mathrm{Zn}$ homeostasis. One important class of such proteins are the metallothioneins (MT), which bind Zn with high affinity but, at the same time, release free $\mathrm{Zn}$ ions in response to 
oxidative/nitrosative stress and thereby modulate the expression of $\mathrm{Zn}$-dependent genes and activate antioxidant enzymes. Differences in $\mathrm{Zn}$ status have also been observed in individuals carrying different alleles for polymorphisms of pro-inflammatory cytokines (i.e. IL-6 and TNF-alpha). In addition, the individual response can be modulated by dietary habits because $\mathrm{Zn}$ absorption and availability is dependent on the intake of other nutrients and trace elements.

These aspects have been poorly studied because the intracellular mechanisms involved in the regulation of $\mathrm{Zn}$ homeostasis are not well understood in the context of aging. Moreover, even if it is known that $\mathrm{Zn}$ can modulate antioxidant responses, very little, if any, focused research aimed at identifying the targets of the intracellular response in ageing has been undertaken. What is known is that during ageing, the intake of $\mathrm{Zn}$ decreases, thus contributing to frailty, general disability and increased incidence of age-related degenerative diseases (cancer, infections and atherosclerosis). This situation may be worsened or ameliorated in different European countries, due to the large differences in extrinsic (dietary habits and socio-economic conditions) and intrinsic factors (genetic background) affecting Zn homeostasis in southern and northern European countries. One of the aims of the Zincage project was to investigate how these factors affect the response to $\mathrm{Zn}$, and thus to contribute to better evaluation of the need for this supplement in elderly populations.

\section{Zn, genetic background and longevity}

Recent advances in understanding molecular and biological processes, evolutionary biology, and epidemiological data strongly suggest that human longevity is the product of genetic, environmental and stochastic interactions. What has been identified as an unfavourable genetic background for the risk of age-related disease and for longevity in some geographic areas is often not confirmed by studies performed in different regions. This suggests that environmental factors strongly interact with the individual genetic background. Different studies on European dietary habits confirm that the intake of micronutrients, including $\mathrm{Zn}$, is different among different European countries. Moreover, the products of some genes with documented polymorphic sites are involved in regulating $\mathrm{Zn}$ homeostasis and inflammatory response, thereby affecting the propensity to develop $\mathrm{Zn}$ deficiency at advanced age. This means that in order to perform appropriate $\mathrm{Zn}$ supplementation in the elderly, it is necessary to take into account the $\mathrm{Zn}$ status, dietary habits and the individual genetic background of each person [2]. This last point may be crucial also in order to identify subjects that are at higher risk to develop Zn deficiency in aging. Here, then, there is potential for a preventive intervention with $\mathrm{Zn}$ supplementation as suggested by Eugenio Mocchegiani
(INRCA-Italian National Research Centres on Aging, Italy), who reported variability in the response to $\mathrm{Zn}$ supplementation according to IL-6-174 and MT1A +647 polymorphisms as well as to country of origin. $\mathrm{Zn}$ supplementation ( $48 \pm 2$ days) at a dose of $10 \mathrm{mg} /$ day as $\mathrm{Zn}$ aspartate (Unizink 50, Kohler Pharma GmbH, Germany) induced a general increase of $\mathrm{Zn}$ levels in the whole supplemented sample ( $\mathrm{n}=148$ elderly), but subjects harbouring different genotypes showed different responses both in the increases of plasma $\mathrm{Zn}$ and the intracellular $\mathrm{Zn}$ status. This finding becomes of paramount importance when we consider the well known role of $\mathrm{Zn}$ homeostasis in regulating inflammatory responses [3]. In fact, according to the antagonistic pleiotropy theory of ageing, natural selection has favoured genes conferring short-term benefits to the organism at the cost of deterioration in later life. Inflamm-aging, the low-grade, chronic, systemic inflammatory state that characterizes the aging process, is just an example of the antagonistic theory as explained by Claudio Franceschi (University of Bologna, Italy). Franceschi reviewed recent advances in understanding the unusual genetics of human longevity, pointing out that a major contribution derives from proinflammatory and anti-inflammatory genes generally associated with age-related diseases [4]. Accordingly, Calogero Caruso (University of Palermo, Italy) reviewed the reports indicating an association between the risk of Alzheimer Disease (AD) and polymorphisms of genes encoding inflammatory mediators, such as IL- $1 \beta$, IL-6, IL10 and TNF- $\alpha$ [5], as well as the possible involvement of $\mathrm{Zn}$. In this respect, the presence of $\mathrm{Zn}$ dyshomeostasis in the $\mathrm{AD}$ brain could be related to the dysregulation of inflammatory mediators in AD patients. However, clear evidence for a possible interplay between dietary $\mathrm{Zn}$ and risk of $\mathrm{AD}$ is still missing.

\section{Zn and brain}

In neurodegenerative diseases, especially $\mathrm{AD}$, a growing literature suggests pathogenic links between $\mathrm{Zn}$ and pathways involved in neurodegeneration. While $\mathrm{Zn}$ supplementation has beneficial antinflammatory effects, it can also play a pivotal role in the polymerization of $\mathrm{A} \beta$ peptide, and plaque formation. Therefore, when supplementating the elderly with $\mathrm{Zn}$, one should always keep in mind that excessive $\mathrm{Zn}$ might have very serious deleterious effects. To address the neurotoxic role of $\mathrm{Zn}$ in neurological diseases, Stefano Sensi (University G. D'Annunzio, Italy) showed how an excessive increase in intracellular free $\mathrm{Zn}^{++}$, either by influx through glutamate ionotropic receptor-associated channels or the mobilization of the cation from MTs and mitochondria, can act as a potent catalytic factor in exacerbating neuronal death in response to excitotoxic levels of glutamate [6]. The excitotoxic cascade by which $\mathrm{Zn}$ exerts its neurotoxicity includes mitochondrial and extra-mitochondrial production of 
reactive oxygen species and disruption of metabolic enzymatic activity, ultimately leading to neuronal necrosis and/or apoptosis. Therefore, not only deficit but also excess of zinc is a condition that may be detrimental, especially for neuronal cells, which are particularly sensitive to oxidative stress. However, the precise role of zinc in $\mathrm{AD}$ and other neurodegenerative diseases is difficult to define because these diseases are progressive, apparently irreversible and fatal, so that it is very difficult to discriminate causal or contributing factors from compensatory defensive mechanisms. Paolo Zatta and Denise Drago (University of Padova, Italy) showed the aggregation kinetic of amyloid-beta after exposure to trace elements (Al, $\mathrm{Zn}$, Fe and $\mathrm{Cu}$ ) [7]. The most dramatic effects were observed with $\mathrm{Al}^{3+}$, and to a lesser extent with $\mathrm{Zn}$, which in turn induced the formation of unstructured filaments which were less toxic than the ones induced by $\mathrm{Al}^{3+}$. Ageing is also associated with a progressive decrease of mitochondrial metabolic competence with the appearance of a kind of mosaicism characterized by increased mitochondria with metabolic impairments or complete metabolic deficiency [8]. Carlo Bertoni (INRCA-Italian National Research Centres on Aging, Italy) illustrated this phenomenon in association with the age-related changes in the synaptic structural dynamics with a particular focus on the mitochondrial targets of $\mathrm{Zn}$. These targets include complexes of the mitochondrial electron transport chain, components of the tricarboxylic acid cycle, and enzymes of glycolysis. An inhibitory effect of $\mathrm{Zn}$ on these targets is most likely, but the relevance of this phenomenon in ageing remains to be investigated appropriately.

\section{Zn and oxidative stress}

A number of $\mathrm{Zn}$-dependent stress-related proteins (PARP1, ApoJ, MT, NO, chaperones) and the activity of Zndependent (SOD) and other (GPX and catalase) antioxidant enzymes in the elderly, as well as the role of the proteasomal system in the degradation of proteins whose abnormal accumulation is harmful in ageing, as well as the role of methionine sulfoxide reductase (Msr) in repairing oxidised proteins were all investigated during the Zincage Project. Alexander Buerkle and Andrea Kunzmann (University of Konstanz, Germany) showed that cellular poly(ADP-ribosyl)ation is positively correlated with $\mathrm{Zn}$ status in human peripheral blood mononuclear cells (PBMC), and confirmed that an age-related decrease of PARP-1 occurs with "in vitro" (T Cell Clone model) and "in vivo" ageing [9]. Zn supplementation "in vitro" increased Poly(ADP-ribosyl)ation in T-cell clones (TCC) and similar effects were obtained after "in vivo" supplementation in elderly people. The influence of $\mathrm{Zn}$ on PARP-1 activity could impact on cellular capacity to repair DNA strand breaks. María Moreno-Villanueva (University of Konstanz, Germany) investigated the effect of exvivo $\mathrm{Zn}$ supplementation on DNA strand break repair, demonstrating that $\mathrm{Zn}$ has a highly significant, positive effect on DNA repair if baseline repair is low (perhaps due to $\mathrm{Zn}$ deficiency). The influence of $\mathrm{Zn}$ on reactive oxygen species (ROS) production in PBMC from healthy elderly subjects, before and after Zn supplementation, was investigated by Jolanta Jajte (Medical University of Lodz, Poland). She found that $\mathrm{Zn}$ may play an important role in lowering oxidative stress in cells of elderly subjects [10], although this effect was not uniform and depended on age, gender and $\mathrm{Zn}$ status before supplementation. Efstathios S. Gonos and Ioannis P. Trougakos (NHRF The National Hellenic Research Foundation, Greece) showed that also the Clusterin/Apolipoprotein J (ApoJ) gene is responsive to $\mathrm{Zn} \mathrm{[11].} \mathrm{MT} \mathrm{and} \mathrm{ApoJ} \mathrm{are} \mathrm{both}$ involved in cell differentiation, so these results may suggest that the $\mathrm{Zn}$ released by MT could affect cell differentiation by affecting ApoJ expression. A positive correlation between ApoJ and $\mathrm{Zn}$ was also shown in the plasma of healthy old subjects and an increasing trend was observed post-supplementation. The origin of the differences in the modulation of ApoJ following supplementation are still being evaluated. The fact that MT can be involved in the regulation of ApoJ as well as other Zn-dependent stressrelated proteins make it very relevant to understand the changes occurring in the function of these proteins with advancing age. Marco Malavolta (INRCA-Italian National Research Centres on Aging, Italy) illustrated the age related changes of these proteins and the interplay between MT protein and mRNA expression as well as their respective modulation by $\mathrm{Zn}$ ions and pro-inflammatory cytokines [12]. MT were found to be associated with ageing, cognitive impairment and perceived stress only when there was reduced $\mathrm{NO}$-induced release of $\mathrm{Zn}$, in other words, in the presence of oxidized and perhaps dysfunctional MT. Therefore, the increased MT observed following Zn supplementation as well as in the presence of good $\mathrm{Zn}$ status, seems a positive sign generally associated with optimal NO-induced release of $\mathrm{Zn}$ and fully functional MT. The hypothesis of dysfunctional MT in ageing was in part confirmed by the results on glutathionylated MT found by Marco Colasanti (University of Rome 3, Italy) [13]. The involvement of these proteins in age-related disease as well as their contribution to longevity, was also confirmed by association studies on MT polymorphisms [14], longevity and cardiovascular disease carried out by Robertina Giacconi and Elisa Muti (INRCA-Italian National Research Centres on Aging, Italy). Other targets of the antioxidant $\mathrm{Zn}$ response are likely to be methionine sulfoxide reductase (Msr) and the proteasome system. Bertrand Friguet and Isabelle Petropoulos (Université Paris 7 Denis-Diderot, France) showed that ageing is associated with a decrease of proteasome chymotrypsin-like peptidase and Msr activities, implicating an impaired response to accumulation of oxidative damage with ageing [15]. In this regard, very relevant results were 
obtained by Zn supplementation in elderly, as shown by Filipe Cabreiro (Université Paris 7 Denis-Diderot, France) in that Zn supplementation improved both proteasome chymotrypsin-like peptidase and Msr activities in the elderly, thus restoring at least in part the intracellular defences against oxidative damage in PBMC [16]. This phenomenon is also evident for chaperone inducibility which, in turn, is markedly reduced in PBMC from old donors [17]. Csaba Sõti and Ákos Putics (Semmelweis University, Hungary) demonstrated that Zn supplementation in elderly subjects induced a marked, significant increase in Hsp72 inducibility upon heat stress. Furthermore, Hsp72 induction displayed a strong correlation with $\mathrm{Zn}$ bioavailability, suggesting that $\mathrm{Zn}$ is a co-inducer of the stress response and a possibly important modulator of the adaptability of the immune system in the elderly. The impact of Zn supplementation on plasma antioxidant enzymes of elderly subjects [18] was studied by Patrizia Mecocci (University of Perugia, Italy). An increased activity of plasma superoxide dismutase and erythrocyte superoxide dismutase, and decreased activity of plasma Cat and GPx were found after supplementation. The reason for such "compensatory" antioxidant enzyme levels is an intriguing clue, perhaps dependent on several factors including genetic background, that will be the object of further investigations. Finally, Wolfgang Maret (University of Texas Medical Branch, TX, USA) reviewed the redox biology of $\mathrm{Zn}$ and $\mathrm{MT}$, focusing on the control of oxidative signalling by the latter [19]. These signals, estimated in picomolar amounts of $\mathrm{Zn}$, appear to be potent effectors. Amplitudes of $\mathrm{Zn}$ signals are determined by the cellular Zn buffering capacity, which is in turn regulated by MT. Therefore, the possible age-associated loss of the ability to make $\mathrm{Zn}$ available for insertion into newly synthesized $\mathrm{Zn}$ metalloenzymes could be related to the agerelated changes in MT and oxidative stress.

\section{Zn and genomic stability}

Some $\mathrm{Zn}$-dependent enzymes involved in the stress response, such as Poly(ADP-ribose) polymerase (PARP1 ), are concerned with the maintenance of telomere function and genomic stability. A fast and reliable highthroughput quantitative method to measure telomere length has been developed by Maria Blasco and Andres Canela (CNIO - Centro Nacional de Investigaciones Oncológicas, Spain), by adapting standard Q-FISH to a 96-well format, coupled with automated microscopy [20]. Applying this method, a significant increment of mean telomere length after Zn supplementation was observed in German donors, whereas Italians displayed no changes. These results confirm that inherited and environmental factors may contribute to affect the response to Zn supplementation. In this respect, specific experiments have been performed by Dawn Mazzatti and Jonathan Powell (Unilever Research Colworth, UK) by applying nutrige- nomic and nutrigenetic approaches to explore the effects of $\mathrm{Zn}$ on gene expression profiles [21] of young and elderly people and to correlate the response in PBMC from elderly subjects to IL-6-174 and MT +647 polymorphisms. At least 13 genes were found to be commonly regulated by $\mathrm{Zn}$ in elderly and young donors (independently of age), whereas most of the other genes were differentially regulated. A very relevant point was the differential regulation of primarily metabolic pathways in the young compared to a mainly inflammatory response in the elderly. However, a subset of the elderly population with MT1A +647 and IL-6-174 polymorphisms (C+C- carriers) responded very favourably to $\mathrm{Zn}$ treatment by reduction in inflammatory cytokine production and altered regulation of energy metabolism. Because these individuals are at high risk for age-related diseases, the anti-inflammatory effects of Zn may represent an important intervention to restore immune function and reduce risk of morbidity and mortality. Jolanta Jajte and Janusz Blasiak (Medical University of Lodz, Poland) studied the impact of $\mathrm{Zn}$ supplementation on DNA damage and DNA repair in Znsupplemented elderly subjects using single cell gel electrophoresis assays (Comet assay) [22]. The overall results suggested that $\mathrm{Zn}$ supplementation may favour the response of the cells to DNA damage, especially in women above 70 years (an age class with a particularly high risk of $\mathrm{Zn}$ deficiency) and that DNA repair processes can be also stimulated by Zn.

\section{Zn and signal triggering}

It is well known that $\mathrm{Zn}$ is involved in the mechanisms of cell cycling, proliferation, cell death and signal transduction, but the modulation of these processes following "in vivo" Zn supplementation in elderly subjects is still an open question. Georges Herbein, Tamas Fulop and Audrey Varin (Université de Franche - Comté, France) investigated IL-6 and IL-2 signalling [23], focusing on the activation of the STAT pathway in T cells of elderly healthy subjects supplemented with $\mathrm{Zn}$. They showed that when subjects with marginal $\mathrm{Zn}$ deficiency were treated with physiological doses of Zn, no changes in Jak/STAT signalling under IL-2 and IL-6 stimulation occur, suggesting that $\mathrm{Zn}$ deficiency was not the underlying cause of the signalling defect and correlating well with previous results on in vitro $\mathrm{Zn}$ supplementation. The presence of large differences due to a different basal Zn status, country of origin, gender and genetic background, could partially explain the lack of a clear homogeneous response. Conversely, $\mathrm{Zn}$ supplementation seemed to have a quite homogeneous effect regarding the modulation of apoptotic mechanisms. Daniela Monti and Rita Ostan (University of Florence, Italy) studied the effect of Zn supplementation on mitochondrial membrane depolarization during spontaneous and deoxyribose-induced apoptosis [24]. Their results indicated a clear protective effect of $\mathrm{Zn}$ both in 
spontaneous and induced apoptosis. However, when the possible protective effect of $\mathrm{Zn}$ was evaluated in relation with polymorphism (codon 72) of the Zn-dependent factor p53, no difference was observed between Pro-and Pro+ carriers, suggesting that the possible protective effect of $\mathrm{Zn}$ on apoptosis is independent of the $\mathrm{p} 53$ polymorphism. No effect was observed in cell cycling before and after $\mathrm{Zn}$ supplementation except for a slight reduction of G2/M phase after Zn supplementation. Further experiments and clinical trials are necessary to clarify the effect of $\mathrm{Zn}$ supplementation in vivo because these encouraging preliminary suggest that this approach could be useful in studying all those diseases with a pathogenesis related to oxidative stress. In particular the involvement of $\mathrm{Zn}$ transporters remains scarcely studied. Nevertheless, it is known that the involvement of $\mathrm{Zn}$ in the mechanisms of apoptosis and cell cycling is not only due to its release from MT but also from the regulation of its influx/efflux via $\mathrm{Zn}$ transporters. Israel Sekler (Ben-Gurion University of the Negev, Israel) described the molecular mechanisms of $\mathrm{Zn}$ homeostasis, with a particular focus on the molecular identity, function and regulation of $\mathrm{Zn}$ transporters [25]. He presented a new hypothesis and results on the regulation of vesicular $\mathrm{Zn}$ transporters and $\mathrm{Na}^{+} / \mathrm{Zn}^{++}$membrane exchange mechanisms which will surely be the object of future research in this field.

\section{Zn and immune mediators}

Long term clonal cultures represent good models for studying the behaviour of T cells under chronic antigenic stress and facilitate the in vitro testing of interventions in a longitudinal ageing system. This reflects the situation in elderly individuals in which $\mathrm{T}$ cells are required to maintain immunity to persistent pathogens, particularly herpes viruses (and especially CMV and EBV), throughout life [26]. These virus-specific T cell clones are sequentially lost during ageing. Longitudinal studies of free-living very elderly people have suggested that such clonal attrition predicts mortality. Jürgen Kempf and Graham Pawelec (University of Tubingen, Germany) showed an increased ability of $\mathrm{T}$ cell clones cultured long-term in certain concentrations of $\mathrm{Zn}$ to synthesise chaperone proteins, following sublethal heat shock. This protective effect of $\mathrm{Zn}$ was even more marked when the cells were challenged with a second, higher, otherwise lethal, heat shock. Thus, if these phenomena also occur in vivo, $\mathrm{Zn}$ supplementation may facilitate protective hormetic events in chronically stressed $\mathrm{T}$ cells and help to prevent the clonal attrition characteristic of the terminal stage of life.

The role of the thymus is vital for orchestration of $\mathrm{T}$ cell development and maturation. Richard Aspinall and Wayne Mitchell (Imperial College London, UK) evaluated the role played by $\mathrm{Zn}$ in maintaining thymic output in healthy supplemented elderly individuals [27]. Analys- ing subjects from different countries, no significant difference was observed in levels of detectable $\mathrm{T}$ cell receptor excision circles (TREC) after Zn supplementation with respect to basal levels. This was found to be the case for both males and females. However, an increasing trend for individuals with particularly low TREC measurements was observed in some countries i.e. males from Italy, Poland and Greece, suggesting that factors affecting individual differences could play a major role. The influence of genetic factors on the immune system was investigated by Erminia Mariani (Istituti Ortopedici Rizzoli, Italy) through the assessment of NK activity and the levels of a pool of pro-inflammatory cytokines and chemokines in elderly subjects [28] before and after Zn supplementation. She reported a general increase of NK cell cytotoxicity after Zn supplementation independent of the country of origin but with differences related to IL-6-174 and MT1A +647 polymorphisms. Regarding the inflammatory profile, it was found to be generally decreased in Italian and German supplemented elderly, whereas it was increased in Greek and Polish subjects. Other than the country of origin, many differences were found when considering individual chemokine and cytokine levels in relation to genetic background, basal Zn status and more general dietary habits. These results confirm that $\mathrm{Zn}$ is a key trace element for a prompt immune response to external noxae. On the other hand, Lothar Rink (RWTH Aachen University, Germany) reported that $\mathrm{Zn}$ inhibited basal proinflammatory cytokine production but stimulated production after antigen stimulation [29]. He also reported a series of beneficial effects of $\mathrm{Zn}$ supplementation observed in the supplemented elderly including reconstituted TH1-cytokine release, decreased spontaneous cytokine production and decreased numbers of activated T cells. All these results were most likely attributed to the increase of intracellular labile $\mathrm{Zn}$ in the PBMC of elderly subjects following the supplementation period. Intracellular labile $\mathrm{Zn}$ could represent an important marker with possible use in clinical diagnosis of $\mathrm{Zn}$ deficiency. The involvement of $\mathrm{Zn}$ transporters in modulating intracellular labile zinc during ageing was also discussed. Finally, a comprehensive review of the essentiality of $\mathrm{Zn}$ for humans was provided by Ananda Prasad (Wayne State University School of Medicine, USA). He described how Zn deficiency affects immune function and illustrated that what can be called a "Zn deficiency syndrome", affects about two billion people [30]. Thus, correction of $\mathrm{Zn}$ deficiency is likely to have a great impact on the health of a large population in the developing world and this could be applicable to the elderly population.

\section{Zn, nutrition and health}

Maternal/fetal nutrition is an important factor in the origin of certain common diseases that affect adults and cause morbidity among the elderly. Contributions of spe- 
cific micronutrient deficiencies to the phenomenon can be inferred. Because Zn deficiency affects at least 1 in 5 people worldwide, and $\mathrm{Zn}$ is essential for many functions [31], not the least of which is the structure/function of thousands of proteins, evidence of the critical importance of $\mathrm{Zn}$ for human pregnancy, and effects of $\mathrm{Zn}$ deficiency during early development in rats on brain maturation and subsequent function in later life was discussed by Harold Sandstead (University of Texas Medical Branch, USA). The essentiality of $\mathrm{Zn}$ for nucleic acid and protein synthesis, and certain epigenetic processes, might explain these experimental findings. Zinc nutriture's specific effects of on epigenetic processes are incompletely understood. Here is the problem of how and how much ageing affects $\mathrm{Zn}$ homeostasis and requirements. Sue J Fairweather-Tait (Institute of Food Research, Norwick, UK) reviewed this aspect showing that the responses to $\mathrm{Zn}$ loading measured as changes in plasma Zn, urinary Zn excretion and liver Zn increase with age while the fraction of $\mathrm{Zn}$ taken up by red blood cells decreased with age. Additionally she illustrated how $\mathrm{Zn}$ absorption is affected by the intake of other nutrients or trace elements [32]. Therefore, a complete evaluation of dietary habits could help to understand the propensity towards $\mathrm{Zn}$ deficiency. The Mediterranean diet has been suggested as a strong modulator of the health status and a determinant of longevity. The assessment of adherence to the Mediterranean diet in European old populations and the investigation of its impact on inflammatory and Zn status was addressed by George Dedoussis (Harokopio University, Greece). He showed that in Greece, where the greatest incidence of obesity among elderly was found [33], as well as in Poland, there is a lower adherence to the Mediterranean diet sometimes associated with a lower $\mathrm{Zn}$ intake than in the rest of Europe. This issue was further reported in detail for the Italian population by Cinzia Giuli and Roberta Papa (INRCA-Italian National Research Centres on Aging, Italy). The relationship between "Zn status" and psycho-social conditions, especially in countries with the highest propensity towards age-related Zn deficiency [34], was illustrated by Fiorella Marcellini (INRCA-Italian National Research Centres on Aging, Italy). She observed also a slight but beneficial effect of $\mathrm{Zn}$ supplementation on cognitive functions of elderly subjects supplemented with Zn. Taking into account that most of these elderly subjects carried specific alleles for IL-6-174 and MT1A +647 polymorphisms, these results further confirm that the response to $\mathrm{Zn}$ intake/supplementation can be affected by the genetic background of each individual.

\section{Conclusion}

$\mathrm{Zn}$ research in the context of ageing is still in a relatively early stage. Several improvements in sensitivity and specificity of methods to assess $\mathrm{Zn}$ status have been developed, but severe limitations in reproducibility still exist.
Nevertheless, new knowledge on the function of metallothioneins and $\mathrm{Zn}$ transporters in ageing has been acquired. Recent results obtained with Zn supplementation in elderly subjects are encouraging. Many factors were found to affect the individual response to $\mathrm{Zn}$, such as general dietary habits, genotype, gender, drug usage and frailty. This makes it very difficult to draw a definitive conclusion regarding the possible benefits of $\mathrm{Zn}$ supplementation during ageing. However, some progress in understanding how $\mathrm{Zn}$ can modulate oxidative stress responses and host defence to infection has been achieved and will hopefully be the subject of further scientific enquiry. It is also clear that in order to clarify many aspects related to the health benefits of $\mathrm{Zn}$, a longitudinal approach will be helpful using cohorts of subjects whose past dietary habits and clinical data can be easily accessed.

\section{Acknowledgements}

Supported by EU Commission (Zincage project, Contract n. FOOD-CT2003-506850, Coordinator Dr. Eugenio Mocchegiani), by INRCA, by TEMA Research Company and BioRad Company.

Zincage Consortium [I]: Eugenio Mocchegiani, Alexander Buerkle, Andrea Kunzmann, Jolanta Jajte, Efstathios S. Gonos, loannis P. Trougakos, Marco Malavolta, Marco Colasanti, Bertrand Friguet, Isabelle Petropoulos, Csaba Soti, Kos Putics, Patrizia Mecocci, Maria Blasco, Andreas Canela, Dawn Mazzatti, Jonathan Powell, Janus Blasiak, Georges Herbein, Tamas Fulop, Audrey Varin, Daniela Monti, Rita Ostan, Graham Pawelec, Richard Aspinall, Wayne Mitchell, Erminia Mariani, Lothar Rink, George Dedoussis, Cinzia Giuli, Roberta Papa, Fiorella Marcellini, Robertina Giacconi, Maria Moreno-Villanueva, Filipe Cabriero, Elisa Muti, Catia Cipriano, Laura Costarelli, Silvia Tesei.

External Speakers: Claudio Franceschi, Calogero Caruso, Paolo Zatta, Denise Drago, Carlo Bertoni-Freddari, Stefano Sensi, Wofang Maret, Israel Sekler, Ananda Prasad, Harold Sandstaed, Sue Fairweather-Tait.

\section{References}

I. [http://www.Zincage.org].

2. Mocchegiani E, Rink L, Blasco M: Zinc and ageing (ZINCAGE Project). Biogerontology 2006, 7:305-6.

3. Vasto S, Mocchegiani E, Malavolta M, Cuppari I, Listi F, Nuzzo D, Ditta $\mathrm{V}$, Candore G, Caruso C: Zinc and inflammatorylimmune response in aging. Ann N Y Acad Sci 2007, I I 00: I I I-22.

4. Franceschi C, Capri M, Monti D, Giunta S, Olivieri F, Sevini F, Panourgia MP, Invidia L, Celani L, Scurti M, Cevenini E, Castellani GC, Salvioli $S$ : Inflammaging and anti-inflammaging: a systemic perspective on aging and longevity emerged from studies in humans. Mech Ageing Dev 2007, I 28:92-105.

5. Candore G, Balistreri CR, Grimaldi MP, Vasto S, Listi F, Chiappelli M, Licastro F, Lio D, Caruso C: Age-related inflammatory diseases: role of genetics and gender in the pathophysiology of Alzheimer's disease. Ann N Y Acad Sci 2006, 1089:472-86.

6. Capasso M, Jeng JM, Malavolta M, Mocchegiani E, Sensi SL: Zinc dyshomeostasis: a key modulator of neuronal injury. J Alzheimers Dis 2005, 8:93-108.

7. Zatta P, Lucchini R, van Rensburg SJ, Taylor A: The role of metals in neurodegenerative processes: aluminum, manganese, and zinc. Brain Res Bull 2003, 62:15-28.

8. Bertoni-Freddari C, Fattoretti P, Giorgetti B, Grossi Y, Balietti M, Casoli T, Di Stefano G, Perretta G: Synaptic and mitochondrial morphometry provides structural correlates of successful brain aging. Ann N Y Acad Sci 2007, 1097:5I-3.

9. Burkle A: DNA repair and PARP in aging. Free Radic Res 2006, 40:1295-302. 
10. Jajte JM: Chemical-induced changes in intracellular redox state and in apoptosis. Int J Occup Med Environ Health 1997, 10:203-212.

II. Trougakos IP, Pawelec G, Tzavelas C, Ntouroupi T, Gonos ES: Clusterin/Apolipoprotein J up-regulation after zinc exposure, replicative senescence or differentiation of human haematopoietic cells. Biogerontology 2006, 7:375-82.

12. Mocchegiani E, Costarelli L, Giacconi R, Cipriano C, Muti E, Rink L, Malavolta M: Zinc homeostasis in aging: two elusive faces of the same "metal". Rejuvenation Res 2006, 9:35I-4.

13. Musci G, Persichini T, Casadei M, Mazzone V, Venturini G, Polticelli $\mathrm{F}$, Colasanti M: Nitrosative/oxidative modifications and ageing. Mech Ageing Dev 2006, I 27:544-5I.

14. Cipriano C, Malavolta M, Costarelli L, Giacconi R, Muti E, Gasparini N, Cardelli M, Monti D, Mariani E, Mocchegiani E: Polymorphisms in MT I a gene coding region are associated with longevity in Italian Central female population. Biogerontology 2006, 7:357-65.

15. Petropoulos I, Friguet B: Maintenance of proteins and aging: the role of oxidized protein repair. Free Radic Res 2006, 40: $1269-76$.

16. Cabreiro F, Picot CR, Friguet B, Petropoulos I: Methionine sulfoxide reductases: relevance to aging and protection against oxidative stress. Ann N Y Acad Sci 2006, 1067:37-44.

17. Soti C, Csermely P: Protein stress and stress proteins: implications in aging and disease. J Biosci 2007, 32:5II-5.

18. Mariani E, Cornacchiola V, Polidori MC, Mangialasche F, Malavolta M, Cecchetti R, Bastiani P, Baglioni M, Mocchegiani E, Mecocci P: Antioxidant enzyme activities in healthy old subjects: influence of age, gender and zinc status: Results from the Zincage Project. Biogerontology 2006, 7:391-8.

19. Maret $\mathrm{W}$ : Zinc coordination environments in proteins as redox sensors and signal transducers. Antioxid Redox Signal 2006, 8: $14|9-4|$.

20. Canela A, Vera E, Klatt P, Blasco MA: High-throughput telomere length quantification by FISH and its application to human population studies. Proc Natl Acad Sci USA 2007, 104:5300-5.

21. Mazzatti DJ, White A, Forsey RJ, Powell JR, Pawelec G: Gene expression changes in long-term culture of T-cell clones: genomic effects of chronic antigenic stress in aging and immunosenescence. Aging Cell 2007, 6:155-63.

22. Blasiak J, Gloc E, Wozniak K, Czechowska A: Genotoxicity of acrylamide in human lymphocytes. Chem Biol Interact 2004, 1 49: 137-49.

23. Fulop T, Larbi A, Douziech N, Levesque I, Varin A, Herbein G: Cytokine receptor signalling and aging. Mech Ageing Dev 2006, I 27:526-37.

24. Ostan R, Alberti S, Bucci L, Salvioli S, Pasi S, Cevenini E, Capri M, Di lorio A, Ginaldi L, De Martinis M, Franceschi C, Monti D: Effect of zinc ions on apoptosis in PBMCs from healthy aged subjects. Biogerontology 2006, 7:437-47.

25. Segal D, Ohana E, Besser L, Hershfinkel M, Moran A, Sekler I: A role for $\mathrm{ZnT}-\mathrm{I}$ in regulating cellular cation influx. Biochem Biophys Res Commun 2004, 323: I 145-50.

26. Pawelec G: Immunity and ageing in man. Exp Gerontol 2006, 4I:1239-42.

27. Mitchell WA, Meng I, Nicholson SA, Aspinall R: Thymic output, ageing and zinc. Biogerontology 2006, 7:46I-70.

28. Mariani E, Cattini L, Neri S, Malavolta M, Mocchegiani E, Ravaglia G, Facchini A: Simultaneous evaluation of circulating chemokine and cytokine profiles in elderly subjects by multiplex technology: relationship with zinc status. Biogerontology 2006, 7:449-59.

29. Haase H, Rink L: Signal transduction in monocytes: the role of zinc ions. Biometals 2007, 20:579-85.

30. Prasad AS: Zinc: mechanisms of host defense. J Nutr 2007, 137:1345-9.

31. Maret W, Sandstead HH: Zinc requirements and the risks and benefits of zinc supplementation. J Trace Elem Med Biol 2006, 20:3-18.

32. Harvey LJ, Dainty JR, Hollands WJ, Bull VJ, Hoogewerff JA, Foxall RJ, McAnena L, Strain J], Fairweather-Tait SJ: Effect of high-dose iron supplements on fractional zinc absorption and status in pregnant women. Am J Clin Nutr 2007, 85: |3|-6.

33. Kanoni S, Dedoussis G, Manios Y, Malavolta M, Mocchegiani E: Health status, blood and anthropometrical indices from Greek old and nonagenarian subjects. Biogerontology 2006, 7:329-37.
34. Marcellini F, Giuli C, Papa R, Gagliardi C, Malavolta M, Mocchegiani E: Psychosocial and biochemical interactions in aging: preliminary results from an Italian old sample of "Zincage" project. Arch Gerontol Geriatr 2007, 44(SuppI I):259-69.
Publish with Biomed Central and every scientist can read your work free of charge

"BioMed Central will be the most significant development for disseminating the results of biomedical research in our lifetime. "

Sir Paul Nurse, Cancer Research UK

Your research papers will be:

- available free of charge to the entire biomedical community

- peer reviewed and published immediately upon acceptance

- cited in PubMed and archived on PubMed Central

- yours - you keep the copyright
BioMedcentral 